\title{
Electrical Substation Reliability Evaluation with Emphasis on Evolving Interdependence on Communication Infrastructure
}

\author{
M.A. Azarm, R. Bari, M. Yue, and Z. Musicki \\ Presented at the 8th International Conference on Probabilistic \\ Method Applied to Power Systems \\ Ames, IA \\ Sept 12-16, 2004 \\ July 2004
Energy Sciences and Technology Department
Brookhaven National Laboratory
P.O. Box 5000
Upton, NY 11973-5000
www.bnl.gov
Managed by
Brookhaven Science Associates, LLC
for the United States Department of Energy under
Contract No. DE-AC02-98CH10886




\section{DISCLAIMER}

This report was prepared as an account of work sponsored by an agency of the United States Government. Neither the United States Government nor any agency thereof, nor any of their employees, nor any of their contractors, subcontractors, or their employees, makes any warranty, express or implied, or assumes any legal liability or responsibility for the accuracy, completeness, or any third party's use or the results of such use of any information, apparatus, product, or process disclosed, or represents that its use would not infringe privately owned rights. Reference herein to any specific commercial product, process, or service by trade name, trademark, manufacturer, or otherwise, does not necessarily constitute or imply its endorsement, recommendation, or favoring by the United States Government or any agency thereof or its contractors or subcontractors. The views and opinions of authors expressed herein do not necessarily state or reflect those of the United States Government or any agency thereof.

\section{FOR UNCLASSIFIED, UNLIMITED STI PRODUCTS}

Available electronically at:

\section{OSTI:}

http://www.osti.gov/bridge

Available for a processing fee to U.S. Department of Energy and its contractors, in paper from:

U.S. Department of Energy

Office of Scientific and Technical Information

P.O. Box 62

Oak Ridge, TN 37831

Phone: (865) 576-8401

Facsimile: (865) 576-5728

E-mail: reports@adonis.osti.gov

\section{National Technical Information Service (NTIS):}

Available for sale to the public from:

U.S. Department of Commerce

National Technical Information Service

5285 Port Royal Road

Springfield, VA 22131

Phone: (800) 553-6847

Facsimile: (703) 605-6900

Online ordering: http://www.ntis.gov/ordering.htm 


\title{
Electrical Substation Reliability Evaluation with Emphasis on Evolving Interdependence on Communication Infrastructure
}

\author{
Mohamad A. Azarm, Robert Bari, Meng Yue, Member, IEEE, Zoran Musicki
}

\begin{abstract}
This study developed a probabilistic methodology for assessment of the reliability and security of electrical energy distribution networks. This included consideration of the future grid system, which will rely heavily on the existing digitally based communication infrastructure for monitoring and protection. Event tree and fault tree methods were utilized. The approach extensively modeled the types of faults that a grid could potentially experience, the response of the grid, and the specific design of the protection schemes. We demonstrated the methods by applying it to a small sub-section of a hypothetical grid based on an existing electrical grid system of a metropolitan area. The results showed that for a typical design that relies on communication network for protection, the communication network reliability could contribute significantly to the frequency of loss of electrical power. The reliability of the communication network could become a more important contributor to the electrical grid reliability as the utilization of the communication network significantly increases in the near future to support "smart" transmission and/or distributed generation.
\end{abstract}

Index Terms - transmission reliability, fault trees, event trees, fault propagation, fault protection.

\section{INTRODUCTION}

T he traditional approach to electrical grid reliability is based on deterministic analyses for congestion and transient response under normal conditions or a condition that satisfies "a single failure criterion". Such methods were shown to be effective and they have resulted in sound designs, which are robust to major single failures. Nevertheless, past events [1][2] have shown that multiple cascading failures under unfavorable conditions have been the major contributor to losses of electrical distribution systems. Another factor in the evolution of the electrical grid systems is their increasing reliance on the digital communication infrastructures for protection and monitoring, which are not easily amenable to traditional deterministic analysis. This reliance is expected to greatly increase and the anticipated communication architectures would be expanded to deal with coordination

M. A. Azarm, R. Bari, and M. Yue are with Energy Science and Technology Department, Brookhaven National Laboratory, Upton, NY 11973-5000 (e-mail: \{azarm\}, \{bari\}, and \{yuemeng\}@bnl.gov).

Z. Musicki is a consultant for Brookhaven National Laboratory (e-mail: musicki@attglobal.net). and control of remote "smart" transmission and/or a future larger population of smaller distributed generators and/or micro-grids.

Powerful risk and reliability methods/tools have been developed over the past three decades [3][4], which were tailored for use in evaluating the reliability of the existing and the future electrical grid system. The general fault tree and event tree analyses were utilized with a set of reliability data to arrive at quantitative system reliability metrics in this study. These methods have the capability of systematically, and in an efficient manner, incorporating the deterministic models for congestion and transient response analyses [5].

The paper is organized as the follows: A general description of the example distribution network including communication infrastructure is given in Section II. The approach and the associated techniques and tools used in this study are presented in Section III. In Section IV, the results are discussed. Conclusion remarks are given in Section V.

\section{SYSTEM DESCRIPTION}

\section{A. Distribution Substation System Description}

The hypothetical system considered for this study is comprised of a main station, which feeds to a number of substations.

Figure 1 presents a simplified block diagram of this hypothetical network. A ring bus configuration of a 345 kilovolts (KV) substation is feeding several smaller areas through $138 \mathrm{KV}$ lines. Two specific substations, namely substations 1 and 2 are considered as a part of this study. The main focus will be on substation 2. Substation 2 consists of five transformer banks feeding two main sync buses in a typical sync bus connection. Operation of two out of these five transformers would be sufficient for carrying the loads. It was estimated that there is approximately 10 hours during a year that three out of five transformers would be required.

Five $138 \mathrm{KV}$ feeder lines feed the five banks of transformers in substation 2 through the associated circuit switchers. The 138-KV circuit switcher will be the primary supply feeder protection and the communication-based ATTT

*This work was performed under the auspices of the U.S. Department of Energy. 
(Audio Tone Transfer Trip) that will be discussed next, is the second independent means of clearing the source for a fault at substation 2. The ATTT system will consist of two ATTT chassis per $138 \mathrm{KV}$ feeder. The operation of two chassis together forms a second line of diverse actuation in the main station (it also trips the transformer at substation 2). The $138 / 13.8 \mathrm{KV}$ transformer in a delta-Y arrangement is used for feeding to standard double synch area station. Each 138/13.8 $\mathrm{KV}$ transformer is protected for transformer differential, zerosequence over voltage protection, reverse current, and the transformer neutral ground differential protection. Each 13.8 $\mathrm{KV}$ feeder is protected for over-current and they are equipped (i.e., a 1200 ohm copper pair resistance regardless of length), an electronic board is typically utilized. Ultimately, the use of transmitters and receivers using ATTT or DCTT will be supplanted in future by direct transfer trip between Relay at Substation 1 communicating with Relay at Substation 2 directly over a digital communication channel or dark fiber.

Figure 2 shows a simplified diagram of an ATTT connection used for the example network being analyzed in this study. It should be noted that two channels of audio tone signals is shown in this figure for simplicity, even though the actual example circuit is composed of five channels for the

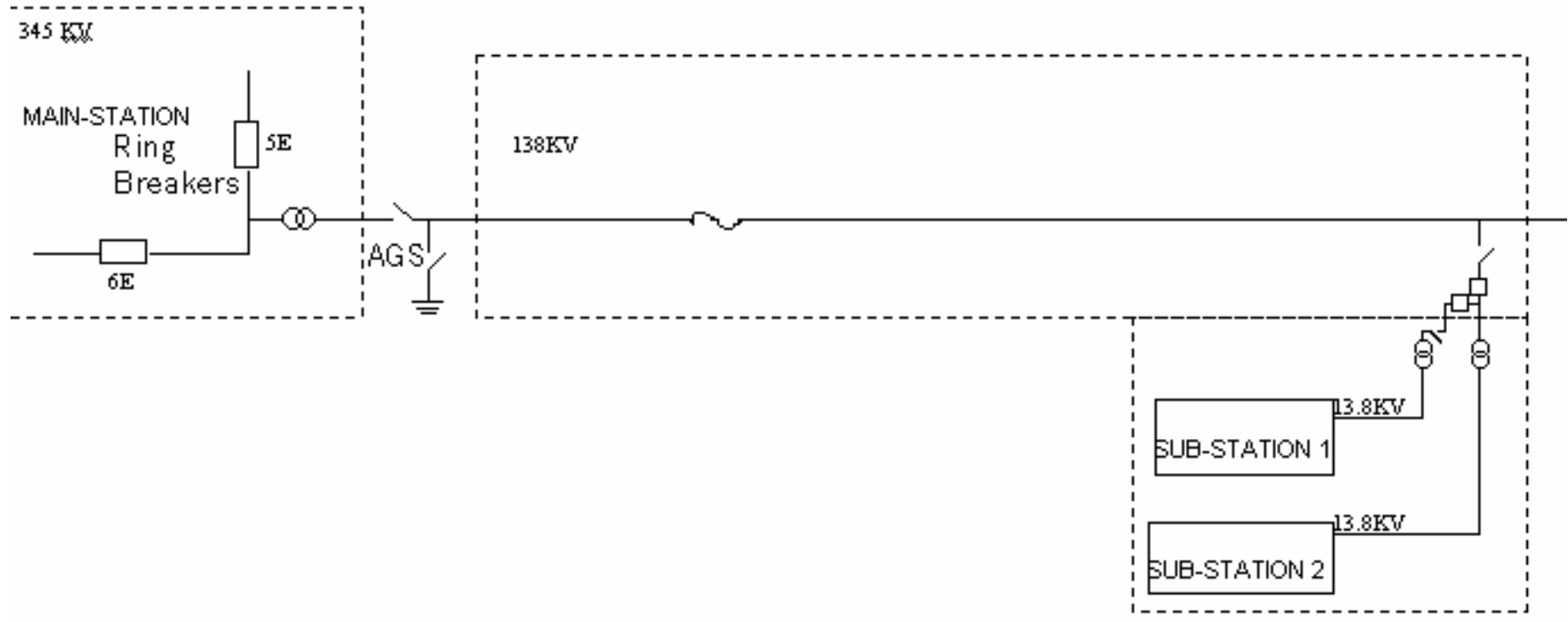

Figure 1 To Level Diagram of Example Connections

with two independent (diverse) relay protections with their communication ports connected to a LAN (Local Area Network).

\section{B. Communication Infrastructure Description}

The actuation trip signals from electrical protection logics are transformed to analog audio tone signals for transmission from one substation to another. The audio tone signal then should be transmitted from one substation to another substation for the purpose of isolation of the faulted zone. The signal transmission could be done by analog means such as modulated wireless connection (e.g. microwave) or digitally through the existing digital communication network infrastructure. The latter is currently considered to be more practical and cost effective due to varying distances and geographic conditions between the two substations.

An alternative to ATTT is through the use of a digitally based pilot wire scheme. In this design, a digital signal representing the DC Transfer Trip (DCTT) is used for remote transmission to other $\mathrm{s}$ for the purpose of differential tripping or monitoring. To regenerate the DC on the pilot wire from the digital signal and to simulate the traditional copper wires five banks of transformers feeding the two synch buses.

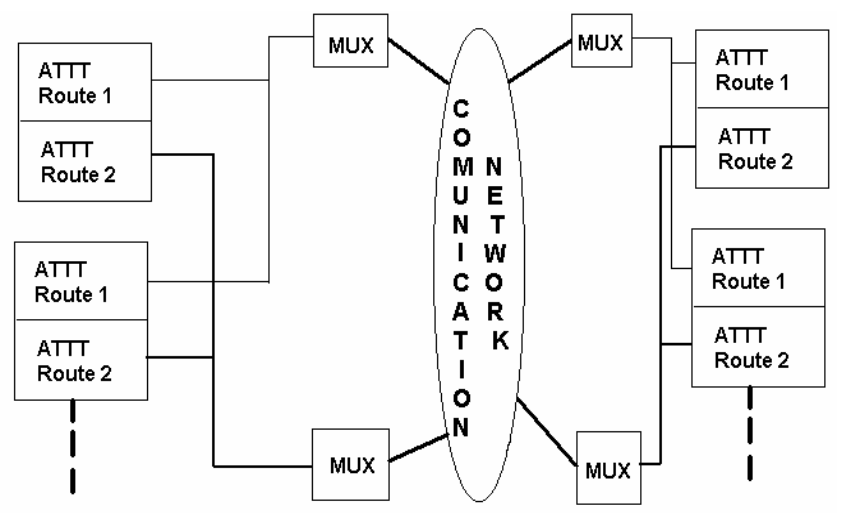

Figure 2 A simplified diagram of ATTT transmission through Communication Network

\section{APPROACH AND TOOLS}

\section{A. Approach}

The electrical grid system has to respond to occasional upset events (also called "initiating events"). Such events 
could be due to an internal fault (e.g., a transformer short circuit), or an external occurrence such as a fire, a flood, a storm, etc. Upset events or "initiating events" are identified here based on experimental data, and/or based on analysis of system failures, which will necessitate actuation of protective features. The initiating events are classified based on their impact on the distribution network. This classification is further refined based on the response of the protective features to the initiator and the impact of the initiator on both the protective features and the network elements. A representative model, albeit conservative, was developed for each class of the initiators. Computerized simulations of the electrical network and the associated protective schemes were sometimes needed to better model the grid response to a class of initiators. This model included the responses of the network, protective devices, consequential isolation or failure of network elements, and potential manual reconfiguration by the operator actions.

The model for each class of initiator is then transformed to a tree-type representation known as event trees. The event trees are the frameworks, which identify all of the pathways in response to each initiating event. The result of each such pathway can be a successful recovery of the system, or partial or complete failure. The various progression paths identified by the event tree are based on the success or the failures of the various functions. Comprehensive decomposition of these functional level progression paths to the constituent basic elements, component, and human actions are done through the development of the fault trees. The failure probabilities associated with these basic elements are provided as input data to the models. All pertinent failure modes are included. The potential for human errors were also included explicitly in both fault trees and event trees when appropriate.

An important failure mode that must be included, because it defeats redundancy protection is common cause failures. There are failures, which may occur at more than one component at once, for example due to improper maintenance, environmental factors, etc. There are several ways of including such failures in the fault trees. Common Cause Failures (CCF) such as improper maintenance, design specification, and environmental factors, which could impact a class of components of similar design, were also modeled within the fault tree structure.

The fault trees are constructed using mostly AND and OR gates, or constructing a Boolean expression of individual failures leading to a higher-level failure. The eventual inputs are component data in the form of probability of failure. All pertinent failure modes are included, e.g., failure to run, failure to start, failure to open, failure to close, spurious actuation, calibration errors, test and maintenance errors, etc. In this study, we mostly deal with failure to open (of the breakers), occasional failure to close, failure to function between test and maintenance, and any calibration errors, which may cause actuation logic to operate improperly.
In summary, the methodology utilized for this study consists of the six major steps. It starts with the identification and classification of initiating events, the identification of protective features, and the analysis and understanding of the grid response. Electrical transient response analyses are sometimes performed to better understand the grid response and identifying the envelopes of failures and success. The approach then follows with the construction of event trees, construction of the fault trees, and gathering of the failure data on all basic events and the initiating event frequency data. Finally, all information collected from all previous steps will be consolidated in a computer model for the purpose of quantification and sensitivity analyses.

\section{B. Tools}

Three types of tools are generally used for such analysis. An integrated fault tree/ event tree analyses code, an integrated electrical transient analysis code, and finally reliability databases and the associated estimation software.

SAPHIRE [4] (Version 7) was utilized as an integrated fault tree/event tree code which is capable of generating all the possible combinations of event causing the undesirable endstate, and estimating the associated occurrence probabilities. SAPHIRE generates and reduces the Boolean logic equations associated with the fault / event tree models to a set of minimal cut sets. The minimal cut sets are then quantified to generate probabilities of the complex events of interest. The probabilities then are decomposed to determine major contributors. Currently, there are other codes with similar capabilities that are commercially available. SAPHIRE is selected since it is available at Brookhaven National Laboratory (BNL) without any charge.

EPTOOL [6] was utilized as an integrated electrical transient analysis code. EPTOOL software was developed at BNL to help simulation of various electrical transients in support of the electrical reliability analyses programs. EPTOOL is an integrated tool for power system and it can perform steady state, dynamic, and transient analyses. EPTOOL was developed using Matlab and Power System Toolbox (PST) 2.0. It consists of following functional blocks:

1. PV curve calculation: computes bus voltage variation with respect to different load levels for a particular power factor on load buses.

2. QV curve calculation: computes reactive power generation variation with respect to different voltage levels on generator or swing buses.

3. Transient analysis/improved transient analysis: simulates the time domain response of a power system for different bus and line outages at different fault clearing time. For the improved transient analysis, the interactions between various protection devices such as loss of synchronism, under-voltage/under-frequency load shedding, and distance relay protection etc. and the system are also 
included. The impacts of the protection and control devices on the power system are simulated in the improved transient analysis. This program can be extended to include more protection and control schemes.

The improved transient analysis of EPTOOL can be used in the reliability evaluation of both transmission and distribution networks. In the case of distribution network, the power sources, the main substations, can be modeled as generator equivalences. The distributed generators are modeled as regular generators. Various protection systems associated with the distributed network can be modeled in EPTOOL. Both permanent and temporary faults are simulated while the protections are enabled accordingly to determine the loss of customer load and/or substations. The simulation results will permit the decision making in event tree/fault tree analysis using SAPHIRE.

4. Small signal analysis: calculates the state space representation of power system at a stable equilibrium point. This feature can be used to develop dynamic control for power systems.

Modal analyses are also available from the small signal analysis block. This function can be further extended to obtain the full system dynamics along a specific trajectory.

BNL's statistical estimation software package including Bayes routine for updating the generic failure rates was also utilized for this study. Generic failure rates from various sources were collected [7] [8] and incorporated to a database. This database is currently limited by scope and it would significantly benefit from incorporating additional data from U.S. electrical utilities and transmission owners. This database also constitutes an important tool for performing such analyses.

\section{RESULTS}

In this study, a failure is defined as a widespread loss of electric power, and a success is defined as an isolation of the electric fault without substantial loss of distribution capacity, e.g., a partial loss of a transformer bank. In this study, we track the progression of an accident from its initiating event, through the response of the protective features of the system, to its appropriate end state.

The failure probability of an undesirable end state, such as loss of the main station, which would result in a widespread loss of electric power to consumers, was considered as the reliability metric of interest. We also calculated the frequency of other end states, which would have lesser impacts.

For the purpose of this study, a fault is simulated in two zones: a fault in a substation, or a fault on the connecting power lines between the main and the substations. Each pathway (combination of protective features failures and successes) identified using event tree technique can be a successful recovery of the system, or partial or complete failure.

The results showed that the frequency of the loss of the main station was on the order of $1 . E-3 / y r$. If we consider all other substations that are typically connected to a main station, and add up the frequencies of all the combinations of similar challenges, the overall frequency of the loss of the main station would rise by about a factor of 20 , or approximately to $2.0 \mathrm{E}-2 / \mathrm{yr}$. This frequency approximately indicates that loss of the main station and experiencing potential widespread power loss to all connected substations is expected, on average, once every 50 years. Similarly, the frequency of the loss of both substations is an order of magnitude lower and it is about 1.E-3 (or once every 1000 years). The low likelihood for loss of both substations was expected since in our hypothetical example, these substations were designed with due considerations for double contingency and adherence to redundancy and diversity.

The study showed that the communication system could significantly contribute to loss of the main station, and, to a lesser degree, to the loss of the substations. Initial results shows that the communication network and the associated lines and the interface cards account for about $26 \%$ of loss of main station and $6 \%$ to loss of substations. These results were obtained when the overall reliability of the communication interface was assumed to be around $99 \%$ (or $1 \%$ unavailability). Sensitivity analysis of the overall unavailability of the communication system and its impact on loss of main station is depicted in Figure 3. As shown in this graph, the overall unavailability for the communication network at 0.1 , the probability of loss of the main station increases by a factor of 3.3 (approximately one loss per 15 years). About $80 \%$ of the probability for loss of main station stem from communication network. Therefore, it would be important to maintain the overall communication system reliability per station at above $99 \%$ to ensure reliable grid performance. Preliminary examination of the experiential data [9] determined that accidents (not routine downtimes) with the capability of affecting more than one central office would become the major issue in maintaining the communication network reliability.

Finally, the study clearly demonstrated that the PRA technology could be utilized for reliability assessment of the electrical grid system. PRA techniques used in this study relied heavily on the specific design and extensively modeled the types of faults that a grid could potentially experience the response of the grid, and the specific design of the protection schemes. The importance of fault detection and protection schemes is heavily emphasized in this methodology. The methodology would systematically identify the cases where supporting electrical transient analyses are required. This would minimize the number of such analyses to the most critical ones. Finally, the methods utilized here are quantitative in nature, thereby allowing prioritization, and the verification that the design will meet the possible reliability 
goals.

\section{Communication Network Sensitivity}

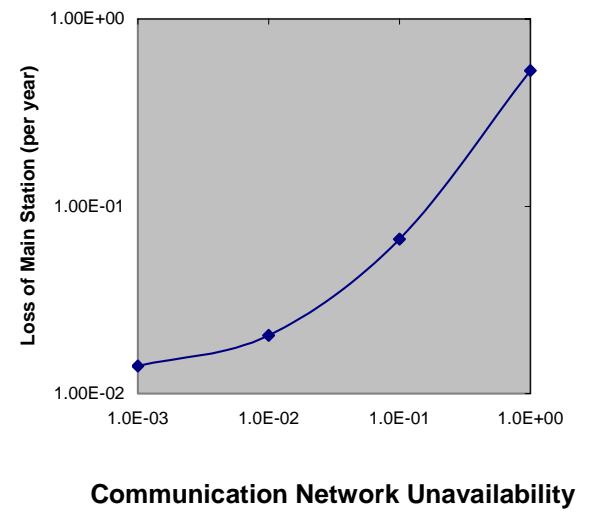

Figure 3: Sensitivity Analysis of Loss of as a Function of Unavailability of Communication Network

\section{CONCLUSIONS}

The reliability assessment method shown in this paper is microscopic in nature and relies heavily on the specific design of the portion of the grid being analyzed. It extensively models the types of faults that a grid could potentially experience, the response of the grid, and the specific design of the protection schemes. The importance of fault detection and protection schemes heavily emphasized and the role of future reliance on the communication infrastructure are addressed. Finally, the method proposed here is quantitative in nature, thereby allowing prioritization and reliability allocation if so desired.

It was shown that the frequency for the loss of the main station is sensitive to the communication network reliability, and it is also sensitive to common cause failure within the electrical network. The reliability of the communication network could become a major contributor to the electrical grid reliability as its utilization significantly increases in the near future. The potential for common cause failures in communication infrastructure, i.e. those events that could affect several central offices, should be eliminated with a high level of confidence to ensure a reliable system. Preliminary examination of the experiential data indicated that non-routine accidents/incidents external to the communication network (not routine unavailability) with the capability of affecting more than one central office would become the major issue in maintaining the communication network reliability.

This study is also considered as a pilot study to show the feasibility of such approaches to evaluate the future gird reliability. Many of the methods and the data utilized are preliminary in nature and should be considered for further development in future.

\section{REFERENCES}

[1] "Review of Selected Electrical System Disturbances in North America," North America Electric Reliability Council, Princeton, New Jersey, March 2001.

[2] “The Washington Heights Network Shutdown - July 6, 1999,” Report by the Corporate Review Committee, Consolidated Edison Company of New York. New York, NY, December 10, 1999.

[3] "Severe Accident Risks for VVER Reactors: Vol. 3: Procedure Guides," NUREG/CR-6572, BNL-NUREG-52534, Brookhaven National Laboratory, September 1999.

[4] Systems Analysis Programs for Hands-on Integrated Reliability Evaluations (SAPHIRE) Version 7.0, Idaho National Engineering \& Environmental Laboratory, Idaho Falls, ID 83415.

[5] M.A. Azarm, R.A. Bari, and Z. Musicki, "Electrical Substation Reliability Evaluation with Emphasis on Evolving Interdependence On Communication Infrastructure,” BNL Technical report (Non-Proprietary Version), Brookhaven National Laboratory, February 2004.

[6] M. Yue, "Power System Analysis Software, Algorithm, Modeling, and User's Guide,” BNL Technical Report, Brookhaven National Laboratory, February 2004.

[7] P.L. Fletcher and W. Degen on behalf of CIGRE Working Group 13.06, "A Summary of the Final Results and Conclusions of the Second International Enquiry on the Reliability of High Voltage Circuit Breakers", Proceedings of the Reliability of Transmission Equipment conference, 29-31 March 1995, Conference Publication No. 406, CIEEE, 1995.

[8] R. Billinton, R. Ghajar, F. Filippelli, and R. Del Bianco, "Transmission Equipment Reliability Using the Canadian Electrical Association Information System", Proceedings of the Reliability of Transmission Equipment Conference, 29-31 March 1995, Conference Publication No. 406, (OIEEE, 1995

[9] NRIC, Network Reliability and Interoperability Council V, Subcommittees 2.A, Focus Group 2, "Network Reliability Best Practices, Packet Switching and Circuit Switching”, Final Report, January 2002 